\section{Fosfomycin, a therapeutic option for infections produced by multiple drug-resistant Enterobacteriaceae}

\author{
Fatemeh Yeganeh Sefidan, ${ }^{1}$ \\ Robab Azargun, ${ }^{1}$ Reza Ghotaslou ${ }^{1,2}$ \\ ${ }^{1}$ Tropical and Infectious Diseases \\ Research Center, ${ }^{2}$ Department of \\ Microbiology, School of Medicine, Tabriz \\ University of Medical Sciences, Tabriz, \\ Iran
}

\section{Abstract}

Due to the increasing prevalence of infections caused by resistant bacteria and especially multiple drug resistance Enterobacteriaceae, availability of alternative effective antibiotics is restricted. The goal of this study was to investigate the susceptibility profile of multiple drug resistance and extensively drug resistance Enterobacteriaceae isolated from various clinical samples to fosfomycin. A total of 303 non-duplicate Enterobacteriaceae isolates were collected. Identification and susceptibility testing were done according to standard microbiological procedures and the Kirby-Bauer test, respectively. Of all isolates, $272(89.8 \%)$ and $26(8.6 \%)$ were detected as multiple drug resistance and extensively drug resistance strains, respectively. The most effective antibiotic (98\%) was fosfomycin, when compared with other antibiotics against multiple drug resistance and extensively drug resistance Enterobacteriaceae isolates. In this study, we find high levels of resistance to commonly used antibiotics. However, fosfomycin can be a good option for treating multiple drug resistance Enterobacteriaceae.

\section{Introduction}

The Enterobacteriaceae is the largest, most diverse group of medically important Gramnegative bacilli and cause a variety of human infections, including septicemia, urinary tract, wound and gastrointestinal infections. ${ }^{1}$ As rapidly as novel antimicrobial agents are introduced, Enterobacteriaceae can develop resistance to antibiotics. Nowadays, a significant increase in antimicrobial resistance of Enterobacteriaceae is observed. ${ }^{2}$ Moreover, the emergence and spread of MDR (multiple drug resistance) and XDR (extensively drug resistance) Enterobacteriaceae, in the community and hospitals, is increasing in the world. ${ }^{3}$ Due to the high rate of antibiotic resistance, selection of antibiotics against MDR bacteria has been limited. ${ }^{4}$ MDR bacteria can transfer the gene to other clinical strains, so the detection of this strain is important. In an era of MDR and XDR, emphasis should be given not only to the development of new antibiotics but also to the reassessment of older and forgotten antimicrobial agents. ${ }^{5}$ Recently, there has been renewed interest in the use of fosfomycin for the management of infections caused by MDR Gram-negative bacteria, mainly Enterobacteriaceae that are resistant to usually used agents. ${ }^{6}$ Fosfomycin is a natural, forgotten antibiotic, known for nearly four decades, broad spectrum and a bactericidal antibacterial agent that inhibits cell wall synthesis in bacteria by inactivating the UDP- $N$-acetyl-glucosamine-3-o-enolpyruvultransferase. ${ }^{6,7}$ There are a few studies on the in vitro activity of fosfomycin against commonly encountered bacteria, except for Escherichia coli and Enterococcus faecalis. ${ }^{8}$ In this regard, the aim of this study was to evaluate the antimicrobial activity of fosfomycin against MDR and XDR Enterobacteriaceae that are resistant to traditional antibiotics.

\section{Materials and Methods}

\section{Bacterial isolates}

A total of 303 non-duplicates Enterobacteriaceae isolates were collected in tertiary care hospitals during February 2014 through August 2015 from 3 cities of Iran; Tabriz, Khoy, and Uremia. Identification of isolates was done by using biochemical tests in the Department of Microbiology, Tabriz University of Medical Sciences, Iran.

\section{Antimicrobial susceptibility testing}

The antimicrobial susceptibility testing was done on Mueller-Hinton agar (Merck, Germany) using Kirby-Bauer's technique according to the Clinical and Laboratory Standards Institute's (CLSI) guidelines ${ }^{8,9}$ The antibiotic discs that were used for determining antimicrobial susceptibility testing were including: ampicillin (10 $\mu \mathrm{g})$, cefazolin (30 $\mu \mathrm{g})$, ceftazidime $(30 \mu \mathrm{g})$, cefepime $(30 \mu \mathrm{g})$, imipenem $(30 \mu \mathrm{g})$, cefuroxime $(30 \mu \mathrm{g})$, aztreonam $(30 \mu \mathrm{g})$, gentamicin $(10 \mu \mathrm{g})$, ciprofloxacin $(5 \mu \mathrm{g})$, tetracycline $(30 \mu \mathrm{g})$, piperacillin/tazobactam (100/10), cotrimoxazole $(30 \mu \mathrm{g})$ and fosfomycin $(200 / 50 \mu \mathrm{g})$ (Mast, Chemical Co, UK). EUCAST has defined fosfomycin zone breakpoints for Enterobacteriaceae (susceptible $\geq 14 \mathrm{~mm}$ and resistant $\leq 13 \mathrm{~mm}$ ).${ }^{10} \mathrm{E}$. coli ATCC 25922 and Pseudomonas aeruginosa ATCC 27,853 strains
Correspondence: Reza Ghotaslou, Department of Microbiology, School of Medicine, Tabriz University of Medical Sciences, Tabriz, Iran.

Tel.: +98.4133364661.

Email: rzgottaslo@yahoo.com

Key words: Enterobacteriaceae; ESBL; fosfomycin; multiple drug resistance; extensively drug resistance.

Acknowledgments: this article was written based on a dataset of Ph. D thesis (number: 93.5-4.8), registered at Tabriz University of Medical Sciences, Tabriz, Iran

Contributions: RG and FYS, study concept and design; RG, analysis and interpretation of data; RA, drafting of the manuscript.

Conflict of interest: the authors declare no potential conflict of interest.

Funding: this project (number: 93-08) was financially supported by Tropical and Infectious Diseases Research Center, Tabriz University of Medical Sciences.

Received for publication: 10 October 2015

Revision received: 9 May 2016.

Accepted for publication: 29 May 2016.

This work is licensed under a Creative Commons Attribution NonCommercial 4.0 License (CC BYNC 4.0).

CC Copyright F. Yeganeh Sefidan et al., 2016

Licensee PAGEPress, Italy

Microbiology Research 2016: 7:6407

doi:10.4081/mr.2016.6407

we used as quality control. In this study, isolates that are resistant to three or more classes of antibiotics are considered MDR. Of these isolates, those that were resistant to entirely but one or two classes of effective antimicrobial agents (not considering fosfomycin) were categorized as extensively drug-resistant (XDR). ${ }^{11}$

Phenotypic screening and confirmatory tests for extended spectrum beta-lactamase (ESBL), 0XA48, class C cephalosporinases (AmpC), Klebsiella pneumoniae Carbapenemase (KPC), and Methalo Beta lactamase (MBL)-production

First screening for ESBL, AmpC and carbapenemase production was carried out based on the disc diffusion agar using ceftazidime $(30 \mu \mathrm{g})$, cefoxitin $(30 \mu \mathrm{g})$, imipenem $(10 \mu \mathrm{g})$ and meropenem $(10 \mu \mathrm{g})$ (Mast, Chemical Co, England) according to the Clinical Laboratory Standards Institute (CLSI) screening criteria for -lactamase production. ${ }^{9}$ Cefoxitin non-susceptible isolates were considered presumptive AmpC-producers. Suspected isolates for $\beta$-lac- 
tamase production were further confirmed using total ESBL/AmpC confirms kit and KPC/MBL and 0XA-48 confirmation kit (Rosco Diagnostica, Denmark). Since the detection of ESBLs can be obscured by chromosomal AmpC producers, ESBL confirmation kit (Rosco Diagnostica, Denmark) was used to distinguish ESBLs in such isolates. Production of KPC and MBL was detected if inhibition zones around meropenem discs containing phenylboronic acid (KPC inhibitor) or dipicolinic acid (MBL inhibitor) were extended by more than 4 and $5 \mathrm{~mm}$, respectively when compared with meropenem disc without inhibitor. Carbapenem resistance related to $\mathrm{AmpC}$ pro- duction couple to decreased permeability was characterized by a $\geq 5 \mathrm{~mm}$, difference in zones between meropenem and meropenem/ cloxacillin discs along with at least a $4 \mathrm{~mm}$ difference between meropenem and meropenem/phenylboronic acid discs. Temocillin nonsusceptible and susceptible isolates, showing negative synergy tests, were identified as OXA48 and porin-deficient ESBL producers, respectively. E. coli ATCC 25922 and Klebsiella pneumonia (K. pneumonia) ATCC 700603 were used as quality control strains in each set of susceptibility tests.

\section{Statistical analyses}

The results were analyzed using SPSS software for Windows (version 17SPSS Inc., Chicago, IL, USA). In this study, $\mathrm{P} \leq 0.05$ was regarded statistically significant.

\section{Results}

The mean age of patients was 52 years old, ranging from 1 to 90 years old, including 125 males and 178 females. The median hospitalization of patients was 6 days. $E$. coli was the

Table 1. Antimicrobial resistance patterns of Enterobacteriaceae to fosfomycin according to source, bacterial isolates, wards, cities, hospitals and other tested antibiotics.

\begin{tabular}{|c|c|c|}
\hline Variables, total (\%) & Susceptible to fosfomycin, n (\%) & Non susceptible to fosfomycin, n (\%) \\
\hline $\begin{array}{l}\text { Type of infection } \\
\text { Urinary, } 219 \text { (72.3) } \\
\text { Bacteremia, } 43(14.2) \\
\text { Burn, } 11(3.6) \\
\text { Respiratory, } 12(4) \\
\text { eritoneal, } 3(1) \\
\text { Meningitis, } 2 \text { (0.7) }\end{array}$ & $\begin{array}{l}213(97.3) \\
43(100) \\
11(100) \\
7(58.3) \\
3(100) \\
2(100)\end{array}$ & $\begin{array}{c}6(2.7) \\
0 \\
0 \\
5(41.7) \\
0 \\
0\end{array}$ \\
\hline $\begin{array}{l}\text { Bacteria } \\
\text { E. coli, } 219(72.3) \\
\text { K. pneumonia, } 57(18.8) \\
\text { E. cloacae, } 14(4.6) \\
\text { P. mirabilis, } 5(1.7) \\
\text { M. morganii, } 2(0.7) \\
\text { K. oxytoa, } 2(0.7) \\
\text { C. freundi, } 1(0.3) \\
\text { P. vulgris, } 2(0.7)\end{array}$ & $\begin{array}{l}217(99.1) \\
56(98.2) \\
13(92.9) \\
5(100) \\
0 \\
2(100) \\
1(100) \\
2(100)\end{array}$ & $\begin{array}{c}2(0.9) \\
1(1.8) \\
1(7.1) \\
0 \\
2(100) \\
0 \\
0 \\
0\end{array}$ \\
\hline $\begin{array}{l}\text { Wards } \\
\text { Internal, } 180 \text { (59.4) } \\
\text { Intensive care unit, } 37 \text { (12.2) } \\
\text { Surgery, } 55 \text { (18.2) } \\
\text { Children, } 18 \text { (5.9) } \\
\text { Burn, } 15 \text { (5) }\end{array}$ & $\begin{array}{l}179(99.4) \\
35(94.6) \\
52(94.5) \\
18(100) \\
15(100)\end{array}$ & $\begin{array}{c}1(0.6) \\
2(5.4) \\
3(5.5) \\
0 \\
0\end{array}$ \\
\hline $\begin{array}{l}\text { Cities } \\
\qquad \begin{array}{l}\text { Tabriz, } 179(89.1) \\
\text { Uremia, } 100(33) \\
\text { Khoy, } 24(7.9)\end{array}\end{array}$ & $\begin{array}{l}176(98.3) \\
97(97) \\
24(100)\end{array}$ & $\begin{array}{c}3(1.7) \\
3(3) \\
0\end{array}$ \\
\hline $\begin{array}{l}\text { Hospitals } \\
\text { Emmam Reza, } 108 \text { (35.6) } \\
\text { Sina, } 71 \text { (23.4) } \\
\text { Emam Khomeini, } 100(33) \\
\text { Qamar, } 9 \text { (3) } \\
\text { Madani, } 15 \text { (5) }\end{array}$ & $\begin{array}{l}105(97.2) \\
71(100) \\
97(97) \\
9(100) \\
15(100)\end{array}$ & $\begin{array}{l}3(2.8) \\
0 \\
3(3) \\
0 \\
0\end{array}$ \\
\hline $\begin{array}{l}\text { Antibiotics resistant } \\
\text { Cefazolin, } 244 \\
\text { Imipenem, } 16 \\
\text { Cefepime, } 112 \\
\text { Ampicillin, } 265 \\
\text { Gentamicin, } 110 \\
\text { Cefuroxime, } 170 \\
\text { Ciprofloxacin, } 172 \\
\text { Cotriomoxazol, } 195 \\
\text { Aztreonam, } 146 \\
\text { Ceftazidime, } 121 \\
\text { Tetracycline, } 118 \\
\text { Piperacillin/tazobactam, } 98\end{array}$ & $\begin{array}{c}238(97.5) \\
15(93.7) \\
110(98.2) \\
261(98.5) \\
107(97.3) \\
166(97.6) \\
169(98.3) \\
192(98.5) \\
144(98.6) \\
119(98.3) \\
115(97.5) \\
95(96.9)\end{array}$ & $\begin{array}{l}6(2.5) \\
1(6.3) \\
2(1.8) \\
4(1.5) \\
3(2.7) \\
4(2.4) \\
3(1.7) \\
3(1.5) \\
2(1.4) \\
2(1.7) \\
3(2.5) \\
3(3.1)\end{array}$ \\
\hline
\end{tabular}


most frequently isolated bacteria (Table 1). According to the results, the highest rate of resistance was in the penicillin group (ampicillin) with $87.5 \%$, followed by the cehems group (cefazolin) with $80.5 \%$, and the folate pathway inhibitors with $64.4 \%$ (Table 2). In contrast, the highest sensitivity rates were discovered in fosfomycin with $98 \%$ and the carbapnem group (imipenem) with $94.7 \%$ (Figure 1). Out of 6 fosfomycin resistant isolates, 5 isolates were isolated from males and 1 was from a female. None of the isolates were sensitive to all antibiotics. The frequency of ESBL, AmpC, KPC and MBL-producing isolates were 112 (36.9\%), 28 (9.2\%), 4 (1.3\%) and 9 (2.9\%) (Table 2). Based on results obtained from susceptibility testing, MDR bacteria was recovered which was 272 out of $303(89.8 \%)$ of the total isolates. Frequency of MDR to three, four, five, six and seven antimicrobial agents were 41 (15.1\%), 52 (19.1\%), 58 (21.3\%), 51 (18.7\%) and 44 (16.2\%), respectively. The XDR was observed in 26 cases $(8.6 \%)$. The most prevalent MDR patterns were resistance to ampicillin, cefazolin, trimethoprim-sulfamethoxazole, cefuroxime and ciprofloxacin. According to results, fosfomycin was also the most effective antibiotic against MDR, XDR, ESBL, AmpC, KPC and MBL-producing isolates.

\section{Discussion}

In the present study, we investigated the susceptibility profile of MDR Enterobacteriaceae isolates to fosfomycin. The main finding of this study is that fosfomycin showed a high in vitro susceptibility to MDR, XDR, CRE, KPC, OXA48 and ESBL producing isolates. In total, $2 \%$ of the MDR and the XDR isolates were resistant to fosfomycin. Numerous studies have distinguished good activity of fosfomycin against MDR Enterobacteriaceae. ${ }^{5,10,12}$ Fairly few studies have assessed the antibacterial activity of fosfomycin against Enterobacteriaceae isolates with XDR, and have provided favorable findings concerning the potential worth of fosfomycin in this regard. $5,11,13$

In comparison to E. coli (0.9\%), K. pneumonia (1.8\%) showed higher rates of resistance to fosfomycin. The low level of resistance to fosfomycin probably is due to limited use of fosfomycin for the treatment of infections in this area. The susceptibility to fosfomycin has not been widely studied for other Enterobacteriaceae. In our study, the majority of isolates were susceptible to fosfomycin; susceptibility rates for $P$. mirabilis, $C$. freundii, $K$. oxytoca and $P$. vulgaris were $100 \%$, except for E. cloacae and $M$. morganii which was detected in $92.9 \%$ and zero, respectively. These results are partly in concordance with previous reports, which indicated that low rate of $E$. coli, $P$. mirabilis and $P$. vulgaris were non-susceptible to fosfomycin. ${ }^{3,14}$ Unfortunately, the spread of ESBL significantly limits the treatment options. The gene encoding the ESBL is located in plasmids and is transmitted among bacteria. These plasmids can carry MDR genes against cotrimoxazole, quinolones and aminoglycosides at the same time. ${ }^{15}$ In the previous studies, $90 \%$ or more of the isolates of Enterobacteriaceae with advanced resistance

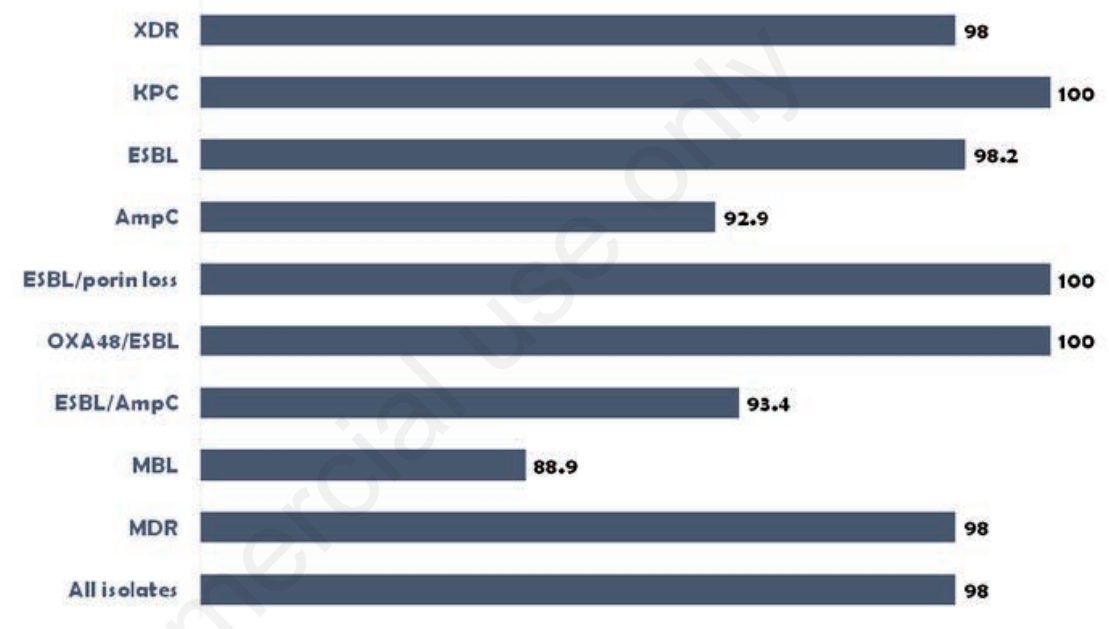

Figure 1. Susceptibility rates (\%) of multiple drug resistance, extensively drug resistance, extended spectrum beta-lactamase, class $\mathrm{C}$ cephalosporinases (AmpC), OXA48, mannanbinding lectin and Klebsiella pneumoniae carbapenemase (KPC)-producing Enterobacteriaceae to fosfomycin.

Table 2. In vitro resistance rates of multiple drug resistance (MDR), extensively drug resistance (XDR), extended spectrum beta-lactamase (ESBLs), class C cephalosporinases (AmpC), mannan-binding lectin (MBL) and Klebsiella pneumoniae Carbapenemase (KPC) producing Enterobacteriaceae isolates to different antibiotics.

\begin{tabular}{|c|c|c|c|c|c|c|c|}
\hline Antibiotics & All isolates & MIDR & XDR & ESBL & AmpC & KPC & MBL \\
\hline Fosfomycin & 2 & 2 & 2 & 1.8 & 7.1 & 0 & 11.1 \\
\hline Cefazolin & 80.5 & 91.7 & 100 & 93.7 & 89.3 & 100 & 100 \\
\hline Ceftazidim & 39.9 & 41.1 & 100 & 81.3 & 50 & 100 & 100 \\
\hline Cefepime & 37 & 44.46 & 98 & 58.9 & 14.3 & 100 & 100 \\
\hline Cefotaxime & 56.1 & 67.5 & 100 & 87.5 & 71.4 & 100 & 100 \\
\hline Aztreonam & 48.2 & 59.3 & 100 & 80.4 & 39.3 & 75 & 77.8 \\
\hline Imipenem & 5.3 & 3.7 & 25 & 10.7 & 7.1 & 100 & 100 \\
\hline Ampicillin & 87.5 & 98 & 100 & 100 & 78.6 & 100 & 100 \\
\hline Gentamicin & 36.3 & 45.7 & 100 & 58.9 & 17.9 & 75 & 100 \\
\hline Ciprofloxacin & 56.8 & 69.5 & 100 & 73.2 & 57.1 & 25 & 88.9 \\
\hline Cotrimoxazol & 64.4 & 77 & 100 & 75.9 & 42.9 & 50 & 100 \\
\hline Tetracycline & 38.9 & 45.7 & 100 & 42 & 39.3 & 25 & 44.4 \\
\hline Piperacillin/Tazobactam & 32.3 & 37 & 75 & 28.6 & 35.7 & 0 & 33.3 \\
\hline
\end{tabular}


to antimicrobial drugs were susceptible to fosfomycin. ${ }^{5,16}$ By contrast, in two studies, ${ }^{10,14}$ fewer than $60 \%$ of the isolates (which were isolates of $E$. aerogenes and $K$. pneumoniae) were susceptible to fosfomycin. $E$. coli seem to be the most susceptible to fosfomycin of the Enterobacteriaceae that create ESBL that in concordance with previous reports. ${ }^{10,14,16}$ However, in the current study, ESBL producing strains showed $98.2 \%$ sensitivity to fosfomycin which concur with reports of previous studies. ${ }^{17,18}$ However fosfomycin is chemically unrelated to other anti-bacterial agents, due to the unique mechanism of action it may provide a synergistic effect with other antibiotics including beta-lactams, aminoglycosides and fluoroquinolone. ${ }^{7}$

While ESBLs are the chief reason of resistance to cephalosporins between Enterobacteriaceae in the world, AmpC betalactamases are emerging as a probable risk to the activity of cephalosporins in many regions. ${ }^{19}$ Cefepime is believed a fourth-generation and frequently effective to $\mathrm{AmpC}$ betalactamases. A new worldwide review of 23,918 isolates from ICUs demonstrated that $74-100 \%$ of isolates were sensitive to cefepime. ${ }^{20}$ In the current study, the most effective antibiotics against AmpC producer Enterobacteriaceae were fosfomycin and imipenem (92.9\%), followed cefepime (85.7\%). Furthermore, fosfomycin and carbapenems are considered extremely effective treatment for AmpC producing infections. ${ }^{21}$

In comparison to tested antibiotics, apart from fosfomycin, imipenem (94.7\%) showed high activity against bacterial isolates. Fosfomycin seems to have retained antibacterial activity against Enterobacteriaceae with progressive resistance patterns, even carbapnem-resistant $K$. pneumoniae. However, CRE is the main threat, and CRE is increasingly common in various parts of the world. ${ }^{14}$ Though, among beta-lactamases, KPC and OXA48 are all carried on plasmids and with no trouble transferred to other isolates. OXA48 enzymes are rising predominantly in the Middle Eastern and European regions and management options are limited. ${ }^{14}$ In the present study, all KPC, AmpC-porin loss, and OXA48 strains were sensitive to fosfomycin. One of the MBL strains was resistant, and 8 were susceptible to fosfomycin. According to results, fosfomycin seems to be a choice antibacterial agent for the management of such difficult to treat infections. Several studies have reported excellent in vitro activity of fosfomycin against CRE isolates. ${ }^{6}$

One important consideration for the clinical use of fosfomycin is the possible for the emergence of resistance during management and for the choice of resistant mutants. ${ }^{6}$ While the natural mutation rate of fosfomycin resistance in Enterobacteriaceae appears to be reasonably high in vitro, this has not commonly been related to the increase of clinically very important fosfomycin resistance in clinical practice. ${ }^{11}$ Nowadays, the oral form of fosfomycin has mainly been used in the management of uncomplicated urinary tract infections in the United States, the United Kingdom, and other countries. However, the intravenous form has been used for indications beyond urinary tract infections in some countries such as Germany, France, Spain and Japan. ${ }^{5}$ The resistance rate to fosfomycin was zero in Enterobacteriaceae isolated from blood, CSF, peritoneal fluid, and burn samples. However, the frequency of resistance to fosfomycin was high (58.3\%) in Enterobacteriaceae isolated from respiratory samples. Current data recommend that fosfomycin might be considered as an option in the management of MDR bacterial infections other than of UTIs, except respiratory infections. The activity of fosfomycin seems to be prone by the site from which the pathogen is isolated. The absence of cross-resistance to fosfomycin with other antibiotics may be attributed to the exclusive mechanism of action of this antimicrobial agent, which includes inhibition of a primary step in bacterial cell wall synthesis. Furthermore, fosfomycin does not seem to be a substrate for common resistance mechanisms of XDR and MDR such as efflux pumps. Moreover, the main type of fosfomycin resistance seems to be chromosomal rather than via plasmid, which reduces the likelihood of co-transmission of resistance to fosfomycin along with resistance to other antibiotics. ${ }^{11}$

In the current study, the disk diffusion assay was used for fosfomycin susceptibility testing. The agar dilution assay is an appropriate method for fosfomycin susceptibility testing, whereas broth dilution tests might give conflicting findings. ${ }^{5}$ Considering that MIC is a time-consuming assay, that several automated systems have not yet included fosfomycin, and that the E-test has shown incompatible results, disc diffusion appears to be the most useful option in routine laboratories to assess susceptibility to fosfomycin. ${ }^{22}$

\section{Conclusions}

In this study, we find high levels of resistance to commonly used antibiotics. The most effective antibiotic is fosfomycin. So, fosfomycin can potentially be considered in the management of infections caused by MDR and XDR Enterobacteriaceae if recognized therapeutic options are not obtainable. Furthermore, fos- fomycin can be included in the routine panel of antibiotics for susceptibility testing by disc diffusion to provide fast and reliable information for the selection of treatment alternatives for MDR and XDR strains.

\section{References}

1. Farajnia S, Alikhani MY, Ghotaslou R, et al. Causative agents and antimicrobial susceptibilities of urinary tract infections in the northwest of Iran. Int J Infect Dis 2009;13:140-4.

2. Tafibakan MI, Tünger A, Ulusoy S. Susceptibility of extended-spectrum betalactamase-producing Escherichia coli urine isolates to fosfomycin, ciprofloxacin, amikacin and trimethoprim-sulfamethoxazole. Turk J Med Sci 2008;38:175-80.

3. Demir T, Buyukguclu T. Evaluation of the in vitro activity of fosfomycin tromethamine against Gram-negative bacterial strains recovered from communityand hospital-acquired urinary tract infections in Turkey. Int $\mathrm{J}$ Infect Dis 2013;17:966-70.

4. Lee SY, Park YJ, Yu JK, et al. Prevalence of acquired fosfomycin resistance among extended-spectrum -lactamase-producing Escherichia coli and Klebsiella pneumoniae clinical isolates in Korea and IS26-composite transposon surrounding fosA3. J Antimicrob Chemother 2012;67:2843-7.

5. Falagas ME, Maraki S, Karageorgopoulos $\mathrm{DE}$, et al. Antimicrobial susceptibility of multidrug-resistant (MDR) and extensively drug-resistant (XDR) Enterobacteriaceae isolates to fosfomycin. Int J Antimicrob Agents 2010;35:240-3.

6. Karageorgopoulos DE, Wang R, Yu X-h, Falagas ME. Fosfomycin: evaluation of the published evidence on the emergence of antimicrobial resistance in Gram-negative pathogens. J Antimicrob Chemother 2012;67:255-68.

7. Michalopoulos AS, Livaditis IG, Gougoutas $\mathrm{V}$. The revival of fosfomycin. Int $\mathrm{J}$ Infect Dis 2011;15:732-9.

8. Lu CL, Liu CY, Huang YT, et al. Antimicrobial susceptibility of commonly encountered bacterial isolates to fosfomycin as determined by the agar dilution and disk diffusion methods. Antimicrob Agents Chemother 2011;55: 4295-301.

9. Clinical and Laboratory Standards Institute (CLSI) (2013) Performance Standards for Antimicrobial Susceptibility Testing; Twenty-Third Informational Supplement. Wayne, PA: CLSI Document 
M100- S23.

10. Liu HY, Lin HC, Lin YC, et al. Antimicrobial susceptibilities of urinary extended-spectrum beta-lactamase-producing Escherichia coli and Klebsiella pneumoniae to fosfomycin and nitrofurantoin in a teaching hospital in Taiwan. J Microbiol Immunol Infect 2011;44:364-8.

11. Falagas ME, Kastoris AC, Kapaskelis AM, Karageorgopoulos DE. Fosfomycin for the treatment of multidrug-resistant, including extended-spectrum -lactamase producing, Enterobacteriaceae infections: a systematic review. Lancet Infect Dis 2010;10:43-50.

12. Neuner EA, Sekeres J, Hall GS, van Duin D. Experience with fosfomycin for treatment of urinary tract infections due to multidrug-resistant organisms. Antimicrob Agents Chemother 2012;56:5744-8.

13. Pontikis K, Karaiskos I, Bastani S, et al. Outcomes of critically ill intensive care unit patients treated with fosfomycin for infections due to pandrug-resistant and extensively drug-resistant carbapenemase-producing Gram-negative bacteria. Int J Antimicrob Agents 2014;43:52-9.

14. Livermore DM, Warner M, Mushtaq S, et al. What remains against carbapenemresistant Enterobacteriaceae? Evaluation of chloramphenicol, ciprofloxacin, colistin, fosfomycin, minocycline, nitrofurantoin, temocillin and tigecycline. Int $\mathbf{J}$ Antimicrob Agents 2011;37:415-9.

15. Qiao LD, Chen S, Yang Y, et al. Characteristics of urinary tract infection pathogens and their in vitro susceptibility to antimicrobial agents in China: data from a multicenter study. BMJ Open 2013;3:004152.

16. Samonis G, Maraki S, Karageorgopoulos D, et al. Synergy of fosfomycin with carbapenems, colistin, netilmicin, and tigecycline against multidrug-resistant Klebsiella pneumoniae, Escherichia coli, and Pseudomonas aeruginosa clinical isolates. Eur J Clin Microbiol Infect Dis 2012;31:695-701.

17. Sultan A, Rizvi M, Khan F, et al. Increasing antimicrobial resistance among uropathogens: Is fosfomycin the answer? Urol Ann 2015;7:26-30.

18. Ho bul T, Ozyurt M, Baylan 0, et al. In vitro activity of fosfomycin trometamol in the treatment of Escherichia coli related uncomplicated urinary tract infections. Mikrobiyol Bul 2009;43:645-9.
19. Harris P, Ferguson J. Antibiotic therapy for inducible AmpC -lactamase-producing Gram-negative bacilli: what are the alternatives to carbapenems, quinolones and aminoglycosides? Int J Antimicrob Agents 2012;40:297-305.

20. Bertrand X, Dowzicky MJ. Antimicrobial susceptibility among gram-negative isolates collected from intensive care units in North America, Europe, the Asia-Pacific Rim, Latin America, the Middle East, and Africa between 2004 and 2009 as part of the Tigecycline Evaluation and Surveillance Trial. Clin Ther 2012;34:12437.

21. Pakyz AL, Oinonen M, Polk RE. Relationship of carbapenem restriction in 22 university teaching hospitals to carbapenem use and carbapenem-resistant Pseudomonas aeruginosa. Antimicrob Agents Chemother 2009;53:1983-6.

22. Pasteran F, Lucero C, Rapoport MJ, et al. Tigecycline and intravenous fosfomycin zone breakpoints equivalent to the EUCAST MIC criteria for Enterobacteriaceae. J Infect Dev Ctries 2012;6:452-6. 